KEK-TH-443

KEK Preprint 95-80

June 22, 2022

$\mathrm{H}$

\title{
Probing the Top-Quark Electric Dipole Moment at a Photon Linear Collider
}

\author{
S.Y. Choi and K. Hagiwara \\ Theory Group, KEK, Tsukuba, Ibaraki 305, Japan
}

\begin{abstract}
We probe the top-quark electric dipole moment (EDM) in top-quark pair production via photon-photon fusion at a photon linear collider. We show how linearly-polarized photon beams can be used to extract information on the topquark EDM without the use of complicated angular correlations of top-quark decay products. If the luminosity of the laser back-scattered photon-photon collisions is comparable to that of the $e^{+} e^{-}$collisions, then the measurement of the top-quark EDM obtained by counting top-quark-production events in photon fusion can be as accurate as the measurement obtained by studying the $t \bar{t}$ decay correlations in $e^{+} e^{-}$collisions using a perfect detector.
\end{abstract}


A detailed study of top-quark physics is one of the most important tasks of a linear $e^{+} e^{-}$collider. The fact that the top quark has a much larger mass than the other quarks and leptons suggests that it may be susceptible to effects from physics beyond the Standard Model (SM), to which lighter quarks and leptons are not sensitive.

Detailed studies have been performed mainly in the processes $e^{+} e^{-} \rightarrow t \bar{t}[\mathbb{1}$, 2] including general studies of $t t \gamma, t t Z$ and $t b W$ couplings [3, 4, 5, 6]. At a photon linear collider we can measure the $t t \gamma$ and $t b W$ couplings. In this paper we investigate the possibility of extracting the effective couplings of the top quark to the photon by employing linearly polarized photons generated by the Compton back-scattering of linearly polarized laser light on electron/positron beams of linear $e^{+} e^{-}$(or $\left.e^{-} e^{-}\right)$colliders.

One may employ two methods to extract the top-quark effective couplings at a photon linear collider. One method makes use of the quasi-freely decaying property of the top quark by measuring various spin correlations in the $t \bar{t}$ final system, $\left(b W^{+}\right)\left(\bar{b} W^{-}\right)$ or $\left(b f_{1} \bar{f}_{2}\right)\left(\bar{b} f_{3} \bar{f}_{4}\right)$. The other method is to employ linearly-polarized photon beams to measure various polarization asymmetries of the initial states. It is, of course, possible to combine the two methods. The former technique is essentially the same as that employed in $e^{+} e^{-}$collisions [3, 4, 5, 6] with one difference; in $e^{+} e^{-}$collisions the spin of the $t \bar{t}$ system is restricted to $J=1$, while in photon fusion $J=0$ or $J \geq 2$ is allowed. Additionally it is easy to produce linearly polarized photon beams through the Compton back-scattering of polarized laser light off the initial electron/positron beams. Hence a $\gamma \gamma$ collider provides some unique opportunities. We will show that CP-odd correlations can be measured by a counting experiment where no information on the momenta and polarization of the top quark decay products are needed.

A CP-odd asymmetry can be formed at a $\gamma \gamma$-collider when the following $J=0$ amplitudes of two photons in the CP-even and CP-odd states are both non-vanishing;

$$
\mathcal{M}[\gamma \gamma \rightarrow X(\mathrm{CP}=+)] \propto \vec{\epsilon}_{1} \cdot \vec{\epsilon}_{2}, \quad \mathcal{M}[\gamma \gamma \rightarrow X(\mathrm{CP}=-)] \propto\left(\vec{\epsilon}_{1} \times \vec{\epsilon}_{2}\right) \cdot \vec{k}_{1},
$$

where $\epsilon_{1}$ and $\epsilon_{2}$ are the polarizations of the two colliding photons and $\vec{k}_{1}$ is the momentum vector of one photon in the $\gamma \gamma$ c.m. frame. This fact has been used in Ref. [7] to show that a Higgs boson $(X=H)$ of mixed CP character could be distinguished from a pure $\mathrm{CP}$ eigenstate by the fact that a certain asymmetry between production cross sections initiated by colliding photons of different helicities is non-zero. A similar analysis can be applied to the $t \bar{t}$ production process $(X=t \bar{t})$ where, for instance, the top-quark EDM generates $\mathrm{CP}$-odd terms. It is evident that the determination of the $\mathrm{CP}$ of $\gamma \gamma$ system requires linearly polarized colliding photons.

In general, a linearly polarized photon state is a superposition of two helicity states with equal weight. The state vector of a linearly polarized photon with the polarization along an azimuthal angle $\phi$ in a given coordinate system can be written as

$$
|\phi\rangle=\frac{1}{\sqrt{2}}\left[-e^{-i \phi}|\lambda=+\rangle+e^{i \phi}|\lambda=-\rangle\right]
$$


The state vector of the two-photon system, where two photons collide head-on, is the direct product

$$
\left|\phi_{1}, \phi_{2}\right\rangle=\frac{1}{2}\left[e^{-i\left(\phi_{1}-\phi_{2}\right)}|++\rangle-e^{-i\left(\phi_{1}+\phi_{2}\right)}|+-\rangle-e^{i\left(\phi_{1}+\phi_{2}\right)}|-+\rangle+e^{i\left(\phi_{1}-\phi_{2}\right)}|--\rangle\right] .
$$

Then the scattering amplitude from the linearly polarized state to a final state $X$ is

$$
\left\langle X|M| \phi_{1}, \phi_{2}\right\rangle=\frac{1}{2}\left[e^{-i\left(\phi_{1}-\phi_{2}\right)} M_{++}-e^{-i\left(\phi_{1}+\phi_{2}\right)} M_{+-}-e^{i\left(\phi_{1}+\phi_{2}\right)} M_{-+}+e^{i\left(\phi_{1}-\phi_{2}\right)} M_{--}\right] .
$$

Here we have used for helicity amplitudes the notation

$$
M_{\lambda_{1} \lambda_{2}}=\left\langle X|M| \lambda_{1} \lambda_{2}\right\rangle
$$

The azimuthal angle difference, $\chi=\phi_{1}-\phi_{2}$, is not changed with respect to rotation along the two-photon direction, while the azimuthal angle sum, $\varphi=\phi_{1}+\phi_{2}$, can be cancelled by an overall rotation along the $z$-axis. After taking an average over the azimuthal angle $\varphi$ we obtain the matrix element squared as

$$
\Sigma(\chi)=\frac{1}{4} \sum_{\lambda_{1}, \lambda_{2}= \pm}\left|M_{\lambda_{1} \lambda_{2}}\right|^{2}-\frac{1}{2} \cos (2 \chi) \operatorname{Re}\left[M_{++} M_{--}^{*}\right]-\frac{1}{2} \sin (2 \chi) \operatorname{Im}\left[M_{++} M_{--}^{*}\right],
$$

which differs from the unpolarized cross section by the last two terms. Only the last term is CP-odd.

We now turn to the specific process $\gamma \gamma \rightarrow t \bar{t}$ with Compton back-scattered laser lights as a photon source at a future linear $e^{+} e^{-}\left(e^{-} e^{-}\right)$collider. The effective Lagrangian for the top-quark-photon interaction is generalized by including terms of dimension five:

$$
\mathcal{L}_{M}=e Q_{t}\left(\frac{d_{t}}{2 m_{t}}\right) \bar{t} \sigma^{\mu \nu} F_{\mu \nu} t, \quad \mathcal{L}_{E}=-i e Q_{t}\left(\frac{\tilde{d}_{t}}{2 m_{t}}\right) \bar{t} \sigma^{\mu \nu} \gamma_{5} F_{\mu \nu} t
$$

where $\sigma^{\mu \nu}=\frac{i}{2}\left[\gamma^{\mu}, \gamma^{\nu}\right], F_{\mu \nu}$ is the photon field strength and $Q_{t}=2 / 3$ is the top-quark electric charge in units of the proton charge $e$. The coefficient $\mu_{t}=e d_{t} Q_{t} / 2 m_{t}$ is then called the anomalous magnetic dipole moment (MDM) of the top quark, which receives one-loop contributions of the order $\alpha_{s} / \pi m_{t}$. This would very likely mask any new-physics contribution to the MDM. On the other hand, the top-quark EDM, $\tilde{\mu}_{t}=e \tilde{d} Q_{t} / 2 m_{t}$, violates T-invariance. Its presence can hence be tested unambiguously via CP-odd correlations. Therefore, we focus in this letter on the measurement of $\tilde{d}_{t}$.

The top-quark EDM modifies the SM $\gamma t \bar{t}$ vertex as

$$
\Gamma^{\mu}\left(k, p, p^{\prime}\right)=-i e Q_{t}\left[\gamma^{\mu}+\tilde{d}_{t} \sigma^{\mu \nu} \gamma_{5} \frac{k_{\nu}}{2 m_{t}}\right]
$$


where $k=p-p^{\prime}$ is the four-momentum of the photon and $p$ and $p^{\prime}$ are four-momenta of the incoming and outgoing top quarks, respectively. With this modification, the helicity amplitudes for the process $\gamma \gamma \rightarrow t \bar{t}$ are in the $\gamma \gamma$ c.m. frame given by

$$
\mathcal{M}_{\lambda_{1} \lambda_{2} ; \sigma \bar{\sigma}}=\frac{4 \pi \alpha Q_{t}^{2} N_{c}}{\left(1-\hat{\beta}^{2} \cos ^{2} \theta\right)} A_{\lambda_{1} \lambda_{2} ; \sigma \bar{\sigma}}
$$

where

$$
\begin{aligned}
& A_{\lambda \lambda ; \sigma \sigma}=-\frac{\sqrt{\hat{s}}}{2 m_{t}}(\lambda+\sigma \hat{\beta})\left[\frac{4 m_{t}^{2}}{\hat{s}}+\tilde{d}_{t}\left(i \lambda-\frac{\tilde{d}_{t}}{2}\right)\left(1+\lambda \sigma \hat{\beta} \cos ^{2} \theta\right)\right], \\
& A_{\lambda \lambda ; \sigma,-\sigma}=\tilde{d}_{t}\left(i \lambda-\frac{\tilde{d}_{t}}{2}\right) \hat{\beta} \cos \theta \sin \theta \\
& A_{\lambda,-\lambda ; \sigma \sigma}=\frac{\sqrt{\hat{s}}}{2 m_{t}} \sigma \hat{\beta} \sin ^{2} \theta\left[\frac{4 m_{t}^{2}}{\hat{s}}-i \tilde{d}_{t} \sigma \hat{\beta}+\frac{\tilde{d}_{t}^{2}}{2}\right], \\
& A_{\lambda,-\lambda ; \sigma,-\sigma}=\hat{\beta}(\lambda \sigma+\cos \theta) \sin \theta\left[1-\frac{\hat{s}}{8 m_{t}^{2}} \tilde{d}_{t}^{2}\left(1-\lambda \sigma \hat{\beta}^{2} \cos \theta\right)\right]
\end{aligned}
$$

where $\lambda, \bar{\lambda}$ and $\sigma / 2, \bar{\sigma} / 2$ are the two-photon and $t, \bar{t}$ helicities, respectively. $\hat{s}$ is the $\gamma \gamma$ c.m. energy squared, $\hat{\beta}=\sqrt{1-4 m_{t}^{2} / \hat{s}}$, and $\theta$ is the polar angle between the photon beam and the top-quark directions. The differential cross section of the process $\gamma \gamma \rightarrow t \bar{t}$ is then given by

$$
\begin{aligned}
\frac{\mathrm{d} \sigma}{\mathrm{d} \cos \theta}\left(\lambda_{1} \lambda_{2} ; \lambda_{1}^{\prime} \lambda_{2}^{\prime}\right) & =\frac{4 \pi \alpha^{2}}{\hat{s}} \frac{Q_{t}^{4} N_{c} \beta}{\left(1-\hat{\beta}^{2} \cos ^{2} \theta\right)^{2}} T_{\lambda_{1} \lambda_{2} ; \lambda_{1}^{\prime} \lambda_{2}^{\prime}}, \\
T_{\lambda_{1} \lambda_{2} ; \lambda_{1}^{\prime} \lambda_{2}^{\prime}} & =\sum_{\sigma_{1} \sigma_{2}= \pm} A_{\lambda_{1} \lambda_{2} ; \sigma_{1} \sigma_{2}} A_{\lambda_{1}^{\prime} \lambda_{2}^{\prime} ; \sigma_{1} \sigma_{2}}^{*}
\end{aligned}
$$

We find to first order in $\tilde{d}_{t}$,

$$
\begin{array}{cc}
\frac{1}{4} \sum_{\lambda_{1} \lambda_{2}= \pm} T_{\lambda_{1} \lambda_{2} ; \lambda_{1} \lambda_{2}}=\frac{1}{2}\left[1+2 \hat{\beta}^{2}-\left(1+\sin ^{4} \theta\right) \hat{\beta}^{4}\right] \\
\operatorname{Re}\left[T_{++;--}\right]=-2\left(1-\hat{\beta}^{2}\right)^{2}, & \operatorname{Im}\left[T_{++;--}\right]=-4\left(1-\hat{\beta}^{2}\right) \operatorname{Re}\left(\tilde{d}_{t}\right) .
\end{array}
$$

One can see from these expressions that, to first order, the electric-dipole-form-factor, $\tilde{d}_{t}$, does not contribute to the unpolarized cross-section, nor to the CP-even term, $\operatorname{Re}\left[T_{++;--}\right]$, but it can contribute to the $\mathrm{CP}$-odd term, $\operatorname{Im}\left[T_{++;--}\right]$. It is now clear that its measurement requires linear polarization of the two photon beams, which can be achieved efficiently via the Compton backscattering of laser beams 8$]$.

The event rate for $t \bar{t}$ production can now be obtained by folding a photon luminosity spectral function, $\mathrm{d} L_{\gamma \gamma}$, with the $t \bar{t}$ production cross section as

$$
\mathrm{d} N=\mathrm{d} L_{\gamma \gamma} \sum_{i, j=0}^{3}\left\langle\zeta_{i} \bar{\zeta}_{j}\right\rangle_{\tau} \sigma_{i j}
$$


where $\zeta_{i}\left(\bar{\zeta}_{i}\right)$ are the Stokes parameters (with $\zeta_{0}=\bar{\zeta}_{0}=1$ ) for $\gamma_{1}\left(\gamma_{2}\right)$, and $\sigma_{i j}$ are the corresponding $t \bar{t}$ production cross-sections. The linear-polarization transfer from the laser photons to the high-energy photons is described by the $\zeta_{1}$ and $\zeta_{3}$ components of the Stokes vector, $\vec{\zeta}=\left(\zeta_{1}, \zeta_{2}, \zeta_{3}\right)$. The length, $l=\sqrt{\zeta_{1}^{2}+\zeta_{3}^{2}}$, of these two components depends on the final state photon energy and on the value of the parameter $x=2 p_{e} \cdot p_{\gamma} / m_{e}^{2}$. $\mathrm{d} L_{\gamma \gamma}$ and $\left\langle\zeta_{i} \bar{\zeta}_{j}\right\rangle_{\tau}$ are obtained as functions of the fraction $\tau(=\hat{s} / s)$ of the $\gamma \gamma$ c.m. energy-squared, $\hat{s}$, to the $e^{+} e^{-}$c.m. energy squared $s$ by averaging over collision, including a convolution over the energy spectra for the colliding photons. Operation below the threshold for $e^{ \pm}$pair production in collisions between the laser beam and the Compton-backscattered photon beam requires $x \leq 2(1+\sqrt{2})$; the lower bound on $x$ depends on the lowest available laser frequency and the production threshold of the final state. The linear-polarization transfer is large for small values of $x$ if the photon energy to electron beam energy, $y$, is close to the maximum value, i.e.

$$
y \rightarrow y_{m}=\frac{x}{1+x}
$$

for a given parameter $x$. The photon energy spectrum is given by

$$
\phi_{0}(y)=\frac{1}{1-y}+1-y-4 r(1-r)
$$

and the Stokes linear component, $l(y)=\phi_{3}(y) / \phi_{0}(y)$, follows from

$$
\phi_{3}(y)=2 r^{2},
$$

with $r=y / x(1-y)$. The maximum value of $l(y)$ is reached at $y=y_{m}$,

$$
l\left(y_{m}\right)=\frac{2(1+x)}{1+(1+x)^{2}}
$$

and approaches unity for small values of $x$. Since only part of the laser polarization is transferred to the high-energy photon beam, it is useful, for the sake of discussion, to define the polarization asymmetry $\mathcal{A}$ as

$$
\mathcal{A}(\tau)=\frac{\left\langle\phi_{3} \phi_{3}\right\rangle_{\tau}}{\left\langle\phi_{0} \phi_{o}\right\rangle_{\tau}}
$$

where

$$
\left\langle\phi_{i} \phi_{i}\right\rangle_{\tau}=\frac{1}{\mathcal{N}^{2}(x)} \int_{\tau / y_{m}}^{y_{m}} \frac{\mathrm{d} y}{y} \phi_{i}(y) \phi_{i}(\tau / y)
$$

The normalization factor $\mathcal{N}(x)$ is defined as

$$
\mathcal{N}(x)=\int_{0}^{y_{m}} \phi_{0}(y) \mathrm{d} y=\ln (1+x)\left[1-\frac{4}{x}-\frac{8}{x^{2}}\right]+\frac{1}{2}+\frac{8}{x}-\frac{1}{2(1+x)^{2}} .
$$


For the linearly-polarized laser beams the number of the $t \bar{t}$ events is given by

$$
\mathrm{d} N=\kappa^{2} L_{e e}\left\langle\phi_{0} \phi_{0}\right\rangle_{\tau} \mathrm{d} \tau\left[\sigma_{0}-\cos (2 \chi) \mathcal{A} \sigma_{A_{1}}-\sin (2 \chi) \mathcal{A} \sigma_{A_{2}} \operatorname{Re}\left(\tilde{d}_{t}\right)\right],
$$

where for the two-photon luminosity function the relation

$$
\mathrm{d} L_{\gamma \gamma}=\kappa^{2} L_{e e}\left\langle\phi_{0} \phi_{0}\right\rangle_{\tau} \mathrm{d} \tau
$$

is used and the respective cross sections are given by

$$
\begin{aligned}
\sigma_{0}(\hat{s}) & =\frac{4 \pi \alpha^{2} Q_{t}^{4} N_{c} \hat{\beta}}{\hat{s}}\left[-2+\hat{\beta}^{2}+\frac{3-\hat{\beta}^{4}}{2 \hat{\beta}} \ln \left(\frac{1+\hat{\beta}}{1-\hat{\beta}}\right)\right], \\
\sigma_{A_{1}}(\hat{s}) & =\frac{4 \pi \alpha^{2} Q_{t}^{4} N_{c} \hat{\beta}}{\hat{s}} \frac{\left(1-\hat{\beta}^{2}\right)}{4}\left[1+\frac{1-\hat{\beta}^{2}}{2 \hat{\beta}} \ln \left(\frac{1+\hat{\beta}}{1-\hat{\beta}}\right)\right], \\
\sigma_{A_{2}}(\hat{s}) & =\frac{4 \pi \alpha^{2} Q_{t}^{4} N_{c} \hat{\beta}}{\hat{s}} \frac{1}{2}\left[1+\frac{1-\hat{\beta}^{2}}{2 \hat{\beta}} \ln \left(\frac{1+\hat{\beta}}{1-\hat{\beta}}\right)\right] .
\end{aligned}
$$

The expression of $\sigma_{0}(\hat{s})$ in Eq. (24) coincides with that of Ref. [9]. The $e-\gamma$ conversion coefficient, $\kappa$, in Eq. (23) is assumed to be 1 in the following analysis. $\chi$ is in the $e^{+} e^{-}$ $\left(e^{-} e^{-}\right)$c.m. frame the angle between the directions of maximum linear polarization of the two laser beams, which are assumed to be approaching nearly head-on towards the electron and positron beams, respectively. And $L_{e e}$ is the integrated $e^{+} e^{-}\left(e^{-} e^{-}\right)$luminosity.

The last term in Eq. (22) depends on $\sin (2 \chi)$ such that the EDM contribution can be isolated by taking the difference of events rates at $\chi=\frac{\pi}{4}$ and $\chi=-\frac{\pi}{4}$. Then one measure of the significance of the EDM contribution is the number of standard deviations $\left(N_{\mathrm{SD}}\right)$ by which $\left|N\left(\chi=\frac{\pi}{4}\right)-N\left(\chi=-\frac{\pi}{4}\right)\right|$ exceeds the expected uncertainty of the background distribution:

$$
N_{\mathrm{SD}}=\operatorname{Re}\left(\tilde{d}_{t}\right) \frac{\sqrt{2} N_{A_{2}}}{\sqrt{N_{0}}}
$$

where

$$
N_{0}=L_{e e} \int_{4 m_{t}^{2} / s}^{\tau_{m}}\left\langle\phi_{0} \phi_{0}\right\rangle_{\tau} \sigma_{0}(\tau s), \quad N_{A_{2}}=L_{e e} \int_{4 m_{t}^{2} / s}^{\tau_{m}}\left\langle\phi_{3} \phi_{3}\right\rangle_{\tau} \sigma_{A_{2}}(\tau s),
$$

with $\tau_{m}=y_{m}^{2}$ in Eq. (15). At the $1-\sigma$ level the allowed maximum value of the EDM parameter, $\operatorname{Re}\left(\tilde{d}_{t}\right)$, is

$$
\left|\operatorname{Re}\left(\tilde{d}_{t}\right)\right|_{m}=\sqrt{\frac{1}{2 N_{0}}} \frac{N_{0}}{N_{A_{2}}},
$$

if no asymmetry is found. The $N_{S D^{-}} \sigma$ level upper bound is obtained simply by multiplying $\left|\operatorname{Re}\left(\tilde{d}_{t}\right)\right|_{m}$ by $N_{\mathrm{SD}}$. We shall evaluate the EDM upper bound, $\left|\operatorname{Re}\left(\tilde{d}_{t}\right)\right|_{m}$ for a yearly integrated luminosity of $20 \mathrm{fb}^{-1}$. 
A crucial experimental issue is the optimal means for maximizing $N_{A_{2}} / \sqrt{N_{0}}$. It requires obtaining the smallest possible value of $x$ to make the linear polarization transfer as large as possible. However, the large top-quark mass does not allow $x$ to be very small. For a given c.m. energy squared, $s$, the allowed range for $x$ is given by

$$
\frac{2 m_{t}}{\sqrt{s}-2 m_{t}} \leq x \leq 2(1+\sqrt{2})
$$

Numerically, for $m_{t}=175 \mathrm{GeV}$ the minimum value of $x$ is $7 / 3$ at $\sqrt{s}=500 \mathrm{GeV}$ and $7 / 13$ at $\sqrt{s}=1 \mathrm{TeV}$, respectively. The EDM upper bound (27) will be very sensitive to the value of $\sqrt{s}$ since a small $x$ requires a large $s$. Fig. 1 shows the cross section $\sigma_{0}$ and the ratio $\sigma_{A_{2}} / \sigma_{0}$ with respect to the $\gamma \gamma$ c.m. energy, $\sqrt{\hat{s}}$, for $m_{t}=150,160,170$, 180 , and $190 \mathrm{GeV}$. The cross section is maximal near $\sqrt{\hat{s}}=2 m_{t}+100 \mathrm{GeV}$, while the ratio $\sigma_{A_{2}} / \sigma_{0}$ is maximal at the threshold. Therefore, it is important to adjust the laser energy and electron/positron beam energies so as to obtain as many $t \bar{t}$ events as possible while retaining $x$ small so as to have large transverse polarization of the back-scattered photons. We make a numerical analysis for $\sqrt{s}=500 \mathrm{GeV}$ and $m_{t}=150 \mathrm{GeV}$ as well as for $m_{t}=170 \mathrm{GeV}$; this facilitates direct comparison with the results of Ref. [5]. Table 1 shows the $x$ dependence of the number of $t \bar{t}$ events, the ratio $N_{A_{2}} / N_{0}$, and the EDM upper bound. $N_{0}$ increases while $N_{A_{2}} / N_{0}$ decreases with $x$. As a result, the optimal upper bound $|\operatorname{Re}(\tilde{\mu})|_{m}$ for $\sqrt{s}=500 \mathrm{GeV}$ is obtained near $x=2.2$ for $m_{t}=150 \mathrm{GeV}$ as

$$
|\operatorname{Re}(\tilde{\mu})|_{m}=\frac{e Q_{t}|\operatorname{Re}(\tilde{d})|_{m}}{2 m_{t}}=5.8 \times 10^{-18}(\mathrm{e} \cdot \mathrm{cm}) .
$$

The 1- $\sigma$ upper bound (29) should be compared with the corresponding bound

$$
|\operatorname{Re}(\tilde{\mu})|_{m}=7.3 \times 10^{-18}(\mathrm{e} \cdot \mathrm{cm}),
$$

which is obtained from the spin correlation study [5] of the cascade process, $e^{+} e^{-} \rightarrow t \bar{t}$, $t \rightarrow b W^{+}\left(\bar{t} \rightarrow \bar{b} W^{-}\right)$, and $W^{-} \rightarrow l \nu_{l}\left(W^{+} \rightarrow l^{+} \bar{\nu}_{l}\right)$ ' for $1.3 \times 10^{4} t \bar{t}$ events expected at $\sqrt{s}=500 \mathrm{GeV}$. It is now clear from the bounds (29) and (30) that, with the $\gamma \gamma$ luminosity comparable to the $e^{+} e^{-}$luminosity, a counting experiment of top-quark pair production events by using linearly polarized laser beams at a photon linear collider can measure the top-quark EDM as accurately as or more accurately than a perfect detector can achieve by studying the $t \bar{t}$ decay correlations in $e^{+} e^{-}$collisions. It is clear that by identifying the $t$ or $\bar{t}$ mometum, we can make use of $J \geq 2$ amplitudes which are large at $\hat{s} \gg m_{t}^{2} 10$.

In summary, it is possible to measure the top-quark EDM in the polarized $\gamma \gamma$ mode by counting $t \bar{t}$ pair production events in a straightforward manner. In the $e^{+} e^{-}$mode it is possible to measure the top-quark EDM by studying $t \bar{t}$ decay correlations. Comparison

\footnotetext{
${ }^{1}$ The $l^{+} l^{-}$channel gives the strongest bound since charged leptons analyze the $t$ and $\bar{t}$ spins most efficiently when the decays are as predicted by the SM.
} 
of our results for the $\gamma \gamma$ mode with previous analyses in the $e^{+} e^{-}$mode shows that the two approaches are competitive. As no information on the momenta and polarization of the top-quark decay products is directly required, linearly-polarized laser beams with an adjustable beam energy provide us with a very efficient way of probing the top-quark EDM at a photon linear collider.

\section{Acknowledgements}

The authors would like to thank R. Szalapski for careful reading the manuscript. This work was supported in part by the Japan Society for the Promotion of Science (No. 94024) and also by the Grant-in-Aid for Scientific Research from the Japanese Ministry of Education, Science and Culture (No. 05228104). 


\section{References}

[1] Proceedings of the 1st International Workshop on "Physics and Experiments with Linear $e^{+} e^{-}$Colliders" (Saariselka, Filand, September 1991), eds. R. Orava, P. Eerola, and M. Nordberg (World Scientific, Singapore, 1992).

[2] Proceedings of the 2nd International Workshop on "Physics and Experiments with Linear $e^{+} e^{-}$Colliders" (Waikoloa, Hawaii, April 1993), eds. F.A. Harris, S.L. Olsen, S. Pakvasa, and X. Tata (World Scientific, Singapore, 1993).

[3] G.L. Kane, G.A. Ladinsky, and C.-P. Yuan, Phys. Rev. D45 (1991) 124.

[4] C.R. Schmidt and M.E. Peskin, Phys. Rev. Lett. 69 (1992) 410; D. Atwood, A. Aeppli and A. Soni, Phys. Rev. Lett. 69 (1992) 2754.

[5] W. Bernreuther and O. Nachtmann, Phys. Rev. Lett. 63 (1989) 2787; W. Bernreuther, O. Nachtmann, P. Overmann and T. Schröder, Nucl. Phys. B388 (1992) 53.

[6] H. Anlauf, W. Bernreuther and A. Brandenburg, PITHA 95/11 (1995).

[7] B. Grazadkowski and J.F. Gunion, Phys. Lett. B294 (1992) 361; M. Krämer, J. Kühn, M.L. Stong and P.M. Zerwas, Z. Phys. C64 (1994) 21.

[8] I.F. Ginzburg, G.L. Kotkin, V.G. Serbo, S.L. Panfil and V.I. Telnov: Nucl. Instr. and Meth. 219 (1984) 5.

[9] J.H. Kühn, E. Mirkes and J. Steegborn, Z. Phys. C57 (1993) 615.

[10] S.Y. Choi and K. Hagiwara, in preparation. 


\section{Tables}

Table 1 The ratio $N_{A_{2}} / N_{0}$, the number of $t \bar{t}$ events $N_{0}$, and the upper EDM bound, $\left[\operatorname{Re}\left(\tilde{\mu}_{t}\right)\right]_{m}$, for selected values of $x$ with $\sqrt{s}=500 \mathrm{GeV}, m_{t}=150 \mathrm{GeV}$ and $L_{e e}=20 \mathrm{fb}^{-1}$. The numbers in parentheses are for $m_{t}=170 \mathrm{GeV}$.

\begin{tabular}{|c|c|c|c|}
\hline$x$ & $N_{0}$ & $N_{A_{2}} / N_{0}$ & $\left|\operatorname{Re}\left(\tilde{\mu}_{t}\right)\right|_{m}\left(10^{-17} \mathrm{e} \cdot \mathrm{cm}\right)$ \\
\hline 1.8 & $156(\cdots)$ & $0.33(\cdots)$ & $0.76(\cdots)$ \\
\hline 2.0 & $395 \quad(\cdots) \mathrm{i}$ & $0.26(\cdots)$ & $0.60(\cdots)$ \\
\hline 2.2 & $680(2)$ & $0.20 \quad(0.31)$ & $0.58 \quad(7.24)$ \\
\hline 2.4 & $983 \quad(36)$ & $0.16 \quad(0.26)$ & $0.60 \quad(2.01)$ \\
\hline 2.6 & 1290 (108) & $0.13(0.21)$ & $0.65 \quad(1.39)$ \\
\hline 2.8 & $1594(206)$ & $0.11 \quad(0.18)$ & $0.71 \quad(1.21)$ \\
\hline 3.0 & $1890(320)$ & $0.09 \quad(0.15)$ & $0.79 \quad(1.16)$ \\
\hline 3.2 & $2178(445)$ & $0.08 \quad(0.13)$ & $0.88(1.16)$ \\
\hline 3.4 & $2456 \quad(574)$ & $0.06 \quad(0.11)$ & $0.98(1.20)$ \\
\hline 3.6 & 2724 (707) & $0.05 \quad(0.09)$ & $1.09(1.27)$ \\
\hline
\end{tabular}

\section{Figures}

Fig. 1 The cross section $\sigma_{0}(\hat{s})$ and the ratio $\sigma_{A_{2}}(\hat{s}) / \sigma_{0}(\hat{s})$ with respect to the $\gamma \gamma$ c.m energy $\sqrt{\hat{s}}$ for $m_{t}=150,160,170,180$, and $190 \mathrm{GeV}$. 
